\title{
3D Characterization of Nano-Pipes and Nano-Pores in Hematite Particles
}

\author{
Niven Monsegue*, Takuya Echigo**, Deepak Bharkhada***, Ge Wang***, Mitsuhiro \\ Murayama*,**** \\ *Institute for Critical Technology and Applied Science, **Department of Geosciences, \\ ***Department of Biomedical Engineering, ****Department of Materials Science \& \\ Engineering; Virginia Polytechnic Institute and State University, Blacksburg VA 24061
}

Scanning transmission electron tomography was used to observe a 3D morphology of hematite nano-particles. In this work, 3D tomography of hematite particles, synthesized by forced hydrolysis of an acidic ferric nitrate solution, display nano-pipes and nano-pores which were not previously resolved by 2D HRTEM images. To study these nano-scale features, careful refinement of tomography tilt series acquisition parameters were carried out; thus, allowing higher STEM magnification to be used during a data set acquisition. Finally, a novel reconstruction algorithm called Interior Tomography (IT) is compared with Weighted Back Projection (WBP) and Serial Iterative Reconstruction Technique (SIRT) in studying the formation of nano-pipes and nano-pores in hematite particles.

Electron tomography was performed using a FEI Titan 80-300 electron microscope operated at $200 \mathrm{kV}$. Tilt-series were acquired in HAADF-STEM mode using a Fischione Model 3000 ADF detector at a beam convergence (half-angle) of $10.5 \mathrm{mrad}$ and a Fischione 2020 ultrahigh-tilt single-axis tomography holder. Images were recorded every $1^{\circ}$ in the tilt range of -65 to $+65^{\circ}$. Hardware calibrations and software parameters were refined to improve tilt series acquisitions at high magnification. These parameters include: defocus, image shift, and specimen holder tilt shift. Once an acquisition of the tilt s eries was completed, images were spatially aligned by cross-correlation algorithm using FEI Inspect 3D Express software. 3D Visualization was performed using FEI Resolve RT.

Figure 1 a. is a representative HRTEM image of a $30 \mathrm{~nm}$ hematite particle showing nanopipes propagating throughout its interior. These nano-pipes are not readily discerned in 2D images. Figure $1 \mathrm{~b}$. shows a SIRT reconstructed rendering of a $30 \mathrm{~nm}$ hematite particle in which a nano-pipe opening is seen on the surface of the hematite particle. For this SIRT reconstruction, a STEM magnification of $\approx 1.8 \mathrm{Mx}$ is used for observing nano-pipes and nano pores in hematite. In comparison, IT will be shown to use a lower magnification yet resolve these internal features.

In order to understand the formation of nano-pipes and nano-pores in hematite, an IT reconstruction algorithm is used and compared to WBP and SIRT. In contrast to WBP and SIRT, IT was developed as a means of accurately reconstructing a region of interest (ROI) within a field of view using X-ray tomography [1]. In this work, IT has been applied to electron tomography for the first time. Its application to electron tomography allows smaller particles (ROI) to be studied at lower magnifications; therefore, overcoming the limitations of particle shifts at high magnifications during sample tilting. Furthermore, with IT it is possible to use fewer projections without substantial image degradation [2]. This makes IT attractive for beam sensitive samples. 
Currently, nano-structured hematite $\left(\alpha-\mathrm{Fe}_{2} \mathrm{O}_{3}\right)$ is considered an exciting and promising material for water-splitting [3], photo-catalyst [4] and solar-cell applications [5] because of the increasing band gap energy by quantum size effect. Also, hematite nano-particles are ubiquitous in natural environments and have many roles of absorbent, redox agent, transporter of heavy/toxic elements [6]. The presence of nano-pores and nano-pipes reported in this work will influence the properties of these particles. For example, nano-pipes and nano-pores will enhance hematite's performance as a catalyst by increasing its surface-area to volume ratio resulting in higher reaction rates. Hence, these nano-scale features are important from both an industrial and environmental aspects. [7]

\section{References}

[1] G. Wang, H. Yu, Can interior tomography outperform lambda tomography?, Proceedings of the National Academy of Sciences of the United States of America, 107 (2010) E92-E93.

[2] Y. Ye, H. Yu, Y. Wei, G. Wang, A general local reconstruction approach based on a truncated Hilbert transform, Journal of Biomedical Imaging, 2007 (2007) 2-2.

[3] A. Kay, I. Cesar, M. Grätzel, New Benchmark for Water Photooxidation by Nanostructured $\alpha^{-}$ $\mathrm{Fe}_{2} \mathrm{O}_{3}$ Films, Journal of the American Chemical Society, 128 (2006) 15714-15721.

[4] D.W. Bahnemann, S.N. Kholuiskaya, R. Dillert, A.I. Kulak, A.I. Kokorin, Photodestruction of dichloroacetic acid catalyzed by nano-sized $\mathrm{TiO}_{2}$ particles, Applied Catalysis B: Environmental, 36 (2002) 161-169.

[5] K. Zhu, T.B. Vinzant, N.R. Neale, A.J. Frank, Removing structural disorder from oriented $\mathrm{TiO}_{2}$ nanotube arrays: reducing the dimensionality of transport and recombination in dye-sensitized solar cells, Nano Letters, 7 (2007) 3739-3746.

[6] M.F.J. Hochella, S.K. Lower, P.A. Maurice, R.L. Penn, N. Sahai, D.L. Sparks, B.S. Twining, Nanominerals, mineral nanoparticles, and Earth systems, science, 319 (2008) 1631-1635.

[7] This work has been supported by the NIH and the Institute for Critical Technology and Applied Science at Virginia Tech.

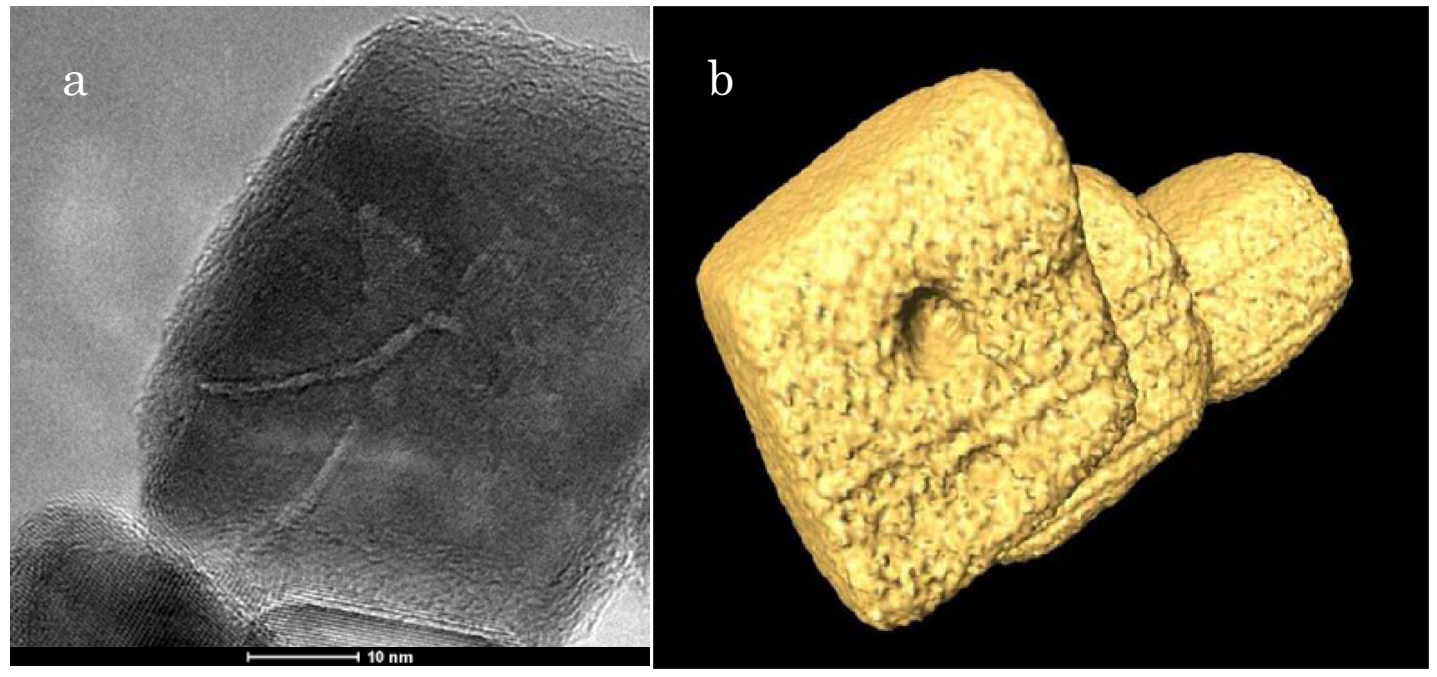

Figure 1 (a) HRTEM images of 30-nm hematite particles showing nano-pipes emerging from the particle's core to its surface. (b) 3-dimensioally reconstructed (SIRT) rendering of 30-nm hematite aggregate displaying a hole on the particle's surface formed by a nano-pipe. 